\title{
Thalamic Glioblastoma: Clinical Presentation, Management Strategies, and Outcomes
}

\author{
Yoshua Esquenazi, MD** \\ Nelson Moussazadeh, MD $^{\S}$ \\ Thomas W. Link, MD $\$$ \\ Koos E. Hovinga, MD \\ Anne S. Reiner, MPH $^{\text {" }}$ \\ Natalie M. DiStefano, BA ${ }^{\ddagger}$ \\ Cameron Brennan, MD ${ }^{\ddagger}$ \\ Philip Gutin, MD \\ Viviane Tabar, MD ${ }^{\ddagger}$
}

\begin{abstract}
*Vivian L. Smith Department of Neurosurgery, The University of Texas Health Science Center at Houston, Houston, Texas; ${ }^{\ddagger}$ Department of Neurosurgery, Memorial Sloan Kettering Cancer Center, New York, New York; ${ }^{\S}$ Department of Neurosurgery, New York Presbyterian Hospital-Weill Cornell Medical Center, New York, New York; "Department of Epidemiology and Biostatistics, Memorial Sloan Kettering Cancer Center, New York, New York
\end{abstract}

\section{Correspondence:}

Yoshua Esquenazi, MD,

Vivian L. Smith Department of

Neurosurgery,

The University of Texas, Health Science

Center at Houston,

6400 Fannin, Suite 2800

Houston, TX 77030.

E-mail:

yoshua.esquenazilevy@uth.tmc.edu

Received, May 3, 2016

Accepted, May 23, 2017.

Published Online, July 21, 2017.

Copyright (C) 2017 by the

Congress of Neurological Surgeons

BACKGROUND: Thalamic glioblastomas (GBMs) represent a significant neurosurgical challenge. In view of the low incidence of these tumors, outcome data and management strategies are not well defined.

OBJECTIVE: To identify the natural history and factors associated with survival in patients with thalamic glioblastoma.

METHODS: A retrospective review of all patients with thalamic glioblastoma over a 10-yr period was performed. Presenting clinical, radiological, and outcome data were collected. Chi-squared and Fisher's exact tests were used to compare clinical characteristics across tumor groups. Cox proportional hazard models were utilized to investigate variables of interest with regard to overall survival.

RESULTS: Fifty-seven patients met inclusion criteria, with a median age of 53 and median Karnofsky Performance Scale (KPS) score of 80 . The most common presenting symptoms were weakness, confusion, and headache. Hydrocephalus was present in $47 \%$ of patients preoperatively. Stereotactic biopsy was performed in 47 cases, and 10 patients underwent craniotomy. The median overall survival was 12.2 mo. Higher KPS, younger age, and cerebrospinal fluid (CSF) diversion were correlated with better overall survival univariately, respectively, while the presence of language deficits at initial presentation was associated with poorer survival. In multivariate analysis, the only significant predictor of survival was presenting KPS.

CONCLUSION: The overall survival of patients with thalamic glioblastoma is comparable to unresectable lobar supratentorial GBMs. Younger patients and those with good presenting functional status had improved survival. Midbrain involvement by the tumor is not a negative prognostic factor. Improved therapies are needed, and patients should be considered for early trial involvement and aggressive upfront therapy.

KEY WORDS: Hydrocephalus, Glioblastoma, Glioma, Thalamus, Ventriculoperitoneal shunt

Neurosurgery 83:76-85, 2018

DOI:10.1093/neuros/nyx349

www.neurosurgery-online.com

G lioblastomas in adults most commonly arise from the cerebral hemispheres and rarely from deep-seated structures such as the diencephalon. As these lesions are considered largely unresectable, resulting in poorer cytoreductive disease control, glioblastoma studies have traditionally excluded patients with deep-seated tumors. ${ }^{1-4}$ Treatment of thalamic glioblastomas (GBMs) is challenging

ABBREVIATIONS: CSF, cerebrospinal fluid; EGFR, epidermal growth factor receptor; GBM, glioblastoma; IRB, Institutional Review Board; KPS, Karnofsky Performance Scale; MRI, magnetic resonance imaging; $\mathbf{O S}$, overall survival due to their deep location within the brain, making them rarely amenable to radical surgical resection. Management strategies range from needle biopsy, to partial or more aggressive resections, and even empiric treatment without histological confirmation. ${ }^{5-7}$ Despite recent evidence that spatial location may dictate the biological behavior of gliomas such as pediatric GBM and diffuse intrinsic pontine glioma, ${ }^{8-10}$ thalamic GBMs have rarely been studied in isolation or in significant numbers. ${ }^{11,12}$ Thus, their natural history remains poorly defined, and treatment paradigms have historically tracked with supratentorial lobar GBM. In this study, we retrospectively evaluated adult patients with thalamic glioblastoma presenting to our 
institution over a 10 -yr period. The goals of this study are to describe the clinical presentation and natural history and to determine prognostic factors that may influence outcome to clarify the behavior and response to treatment of this rare subset of tumors. ${ }^{13,14}$

\section{METHODS}

An Institutional Review Board (IRB)-approved, retrospective chart review of all adult patients with pathologically confirmed thalamic glioblastoma at our institution between 2005 and 2015 was performed. Additional patient consent was not sought per the standards of practice outlined by our institution. Patients were identified via a search of a prospectively collected brain tumor registry database, and all tumors affecting the thalamus and adjacent structures were included in the study. Because in many cases it was challenging to define whether the main bulk of the tumor had arisen from the thalamus or other adjacent structures, all tumors affecting the thalamus were included. Tumors involving only basal ganglia, midbrain, hypothalamus, optic pathways, pineal region, or ventricles without thalamic involvement; patients with previous histologically confirmed lower grade gliomas; and patients younger than age 18 were excluded. Diagnosis of glioblastoma was made by a neuropathologist according to the World Health Organization classification system. ${ }^{15}$ Clinical, radiological, and outcome data were collected from medical records, telephone interviews, and the Social Security Death Index. Compiled data fields included demographic data, Karnofsky Performance Scale (KPS) attributes, presenting symptoms, preoperative tumor magnetic resonance imaging (MRI) characteristics, surgical intervention with extent of resection, histopathologic and molecular data, and adjuvant therapy. Outcome data included postoperative surgical complications, need for cerebrospinal fluid (CSF) diversion, and overall survival (OS). Patients whose deaths were unconfirmed were censored at the time of their last clinic encounter.

Follow-up imaging, when available, was used to evaluate patterns of tumor progression. In patients undergoing craniotomy, the degree of resection was classified as gross total when no residual enhancement was noted on MRI, near total if only rim enhancement of the resection cavity was noted, or subtotal if residual nodular enhancement was noted, in accordance with previously published conventions. ${ }^{3}$ Volumetric measurements were obtained using iPlan Net 3.0.0 software (BrainLAB AG, Germany). Regions of interest were manually drawn on each postcontrast axial T1-weighted image and used to compute the volume of the tumor in cubic centimeters. For tumors with no or minimal enhancement, axial T2-Flair images were used for analysis.

\section{Statistical Analysis}

Descriptive statistics such as frequencies, medians, means, and standard deviations were used to characterize the population under study. OS was calculated from surgery until death or last follow-up. Cox proportional hazard models were utilized to univariately investigate variables of interest with regard to OS. Variables that reached a significance level of $\alpha<0.05$ univariately were included in a multivariate Cox proportional hazards model. Results of interest were graphically presented with Kaplan-Meier curves. Comparisons of categorical variables by shunt status at presentation were performed using Fisher's exact test or chisquared test where appropriate. Comparisons of continuous variables by shunt status at presentation were performed using the Wilcoxon rank sum test. All $P$ values were 2 -sided with a level of significance less than
TABLE 1. Population Demographics and Clinical Characteristics and Presentation of Thalamic Glioblastomas at Memorial Sloan Kettering Cancer Center

\begin{tabular}{|lccc|}
\hline Variable & Level & $\mathbf{n}$ & $\%$ \\
\hline Location & Bilateral & 3 & 5 \\
Hydro & Left & 25 & 44 \\
& Right & 29 & 51 \\
Hemorrhagic & No & 30 & 53 \\
Seizures & Yes & 27 & 47 \\
Headache & No & 52 & 91 \\
& Yes & 5 & 9 \\
N/V & No & 53 & 93 \\
Motor symptoms & Yes & 3 & 7 \\
Sensory symptoms & No & 30 & 53 \\
Language symptoms & Yes & 27 & 47 \\
& No & 44 & 77 \\
Visual symptoms & Yes & 13 & 23 \\
& No & 22 & 39 \\
Confusion & Yes & 35 & 61 \\
& No & 37 & 65 \\
Memory loss & Yes & 20 & 35 \\
Midbrain & No & 45 & 79 \\
Gender & Yes & 12 & 21 \\
& No & 43 & 75 \\
& Yes & 14 & 25 \\
& No & 29 & 51 \\
& Yes & 28 & 49 \\
& No & 33 & 58 \\
& Yes & 24 & 42 \\
& No & 39 & 68 \\
& Yes & 18 & 32 \\
& Male & 26 & 46 \\
& & 31 & 54 \\
\hline
\end{tabular}

.05. All statistical analyses were performed in SAS version 9.4 (SAS Institute Inc, Cary, North Carolina).

\section{RESULTS}

\section{Patient Demographics and Clinical Presentation}

Fifty-seven patients ( 31 men and 26 women), ranging from 18 to $83 \mathrm{yr}$ in age (median $53 \mathrm{yr}$ ), were included. Forty-nine $(86 \%)$ patients underwent initial biopsy/craniotomy at outside institutions and referred to our center for further management. Eight patients underwent surgical intervention (stereotactic needle biopsy) at our center. Median preoperative KPS for all patients was 80 , and presenting symptoms included hemiparesis $(61 \%)$, confusion $(49 \%)$, headache $(47 \%)$, memory loss $(42 \%)$, nausea/emesis $(23 \%)$, and seizure $(7 \%)$. The median preoperative tumor volume was $13 \mathrm{~cm}^{3}$ (range 0.14-69.75). Twenty-nine tumors were right-sided, 25 were left-sided, and 3 bilateral. Motor (weakness), sensory (numbness/tingling), language (naming difficulties, apraxia, and slowness of speech), and visual (blurry/double vision) symptoms were found in 35, 20, 12, and 14 patients, respectively. All but one of the patients presenting with language 
TABLE 2. Cox Regression for Overall Survival of Thalamic Glioblastomas at Memorial Sloan Kettering Cancer Center

\begin{tabular}{|c|c|c|c|c|c|c|c|c|c|}
\hline Variable & Level & $\mathbf{n}$ & $\%$ & \multicolumn{3}{|c|}{ Univariate } & \multicolumn{3}{|c|}{ Multivariate } \\
\hline Location & Right & 29 & 54 & ref & - & - & & & \\
\hline \multirow[t]{2}{*}{ Surgery } & Biopsy & 47 & 82 & ref & - & - & & & \\
\hline & Craniotomy & 10 & 18 & 0.94 & $0.47-1.88$ & .86 & & & \\
\hline Hydro & No & 30 & 53 & ref & - & - & & & \\
\hline Headache & Yes & 27 & 47 & 0.69 & $0.39-1.23$ & .21 & & & \\
\hline \multirow[t]{2}{*}{ N/V } & No & 44 & 77 & ref & - & - & & & \\
\hline & Yes & 13 & 23 & 1.18 & $0.60-2.33$ & .63 & & & \\
\hline \multirow[t]{2}{*}{ Motor symptoms } & No & 22 & 39 & ref & - & - & & & \\
\hline & Yes & 35 & 61 & 1.63 & $0.89-2.98$ & .12 & & & \\
\hline Sensory symptoms & No & 37 & 65 & ref & - & - & & & \\
\hline Visual symptoms & Yes & 14 & 25 & 0.79 & $0.42-1.50$ & .47 & & & \\
\hline \multirow[t]{2}{*}{ Confusion } & No & 29 & 51 & ref & - & - & & & \\
\hline & Yes & 28 & 49 & 1.03 & $0.59-1.80$ & .93 & & & \\
\hline \multirow[t]{2}{*}{ Memory loss } & No & 33 & 58 & ref & - & - & & & \\
\hline & Yes & 24 & 42 & 1.01 & $0.58-1.77$ & .97 & & & \\
\hline \multirow[t]{2}{*}{ VPS } & No & 37 & 65 & ref & - & - & ref & - & - \\
\hline & Yes & 20 & 35 & 0.43 & $0.23-0.80$ & .008 & 0.58 & $0.29-1.13$ & .11 \\
\hline \multirow[t]{2}{*}{ Midbrain } & No & 39 & 68 & ref & - & - & & & \\
\hline & Yes & 18 & 32 & 0.86 & $0.48-1.57$ & .63 & & & \\
\hline \multirow[t]{2}{*}{ Gender } & Female & 26 & 46 & ref & - & - & & & \\
\hline & Male & 31 & 54 & 1.18 & $0.67-2.08$ & .58 & & & \\
\hline Volumetrics & - & - & - & 1.01 & $0.99-1.03$ & .22 & & & \\
\hline
\end{tabular}

symptoms had left-sided tumors. Confusion and memory loss were found in 28 and 24 patients, respectively (Table 1). Twentyseven cases had molecular profiling data available: 1 of 12 were IDH1 mutant (8\%) and 0 of 5 harbored epidermal growth factor receptor (EGFR) variant III. Nine of 13 cases were EGFR amplified (69\%, notably not including the IDH1 mutant case), and 7 of 9 were MGMT unmethylated (78\%). The IDH mutant case was EGFR wild type, unamplified and MGMT unmethylated. Immunohistochemistry for histone H3-K27M mutant protein was performed only on 4 patients in whom available tissue was available. There was no convincing evidence of the mutation in any of the 4 patients.

\section{Management}

Management strategies are outlined in Table 2. Stereotactic biopsy was performed in 47 cases and craniotomy in 10 (7 transcortical and 3 interhemispheric transcallosal approaches) patients. Postoperative imaging in patients undergoing craniotomy demonstrated subtotal resection in all cases. Of
8 patients sampled at our institution (14\%), all underwent stereotactic needle biopsy. Among 27 patients with hydrocephalus, 2 required emergent external ventriculostomy placement upon presentation, and 20 required sustained CSF diversion via ventriculoperitoneal shunting, one of whom had failed prior endoscopic third ventriculostomy. Immediate postoperative surgical complications occurred in 9 patients (16\%): 7 (15\%) suffered fixed neurological deficits following stereotactic biopsy (worsened hemiparesis in 5, hemiplegia in 1, and visual field decline in 1), and 2 cases of asymptomatic intratumoral hemorrhage occurred. New permanent deficits occurred in 2 of 10 (20\%) patients undergoing craniotomy (1 paresis, 1 plegia). Fifty patients received adjuvant temozolamide and conventional external radiation therapy $(88 \%)$. Three patients were referred to hospice early in their diagnosis and did not receive adjuvant therapy. Thirteen patients received bevacizumab $(22 \%)$ at recurrence. Data regarding duration of bevacizumab treatment was available on 12 patients (median 3 mo). Records from outside institutions for adjuvant therapy on 4 patients were not available. 


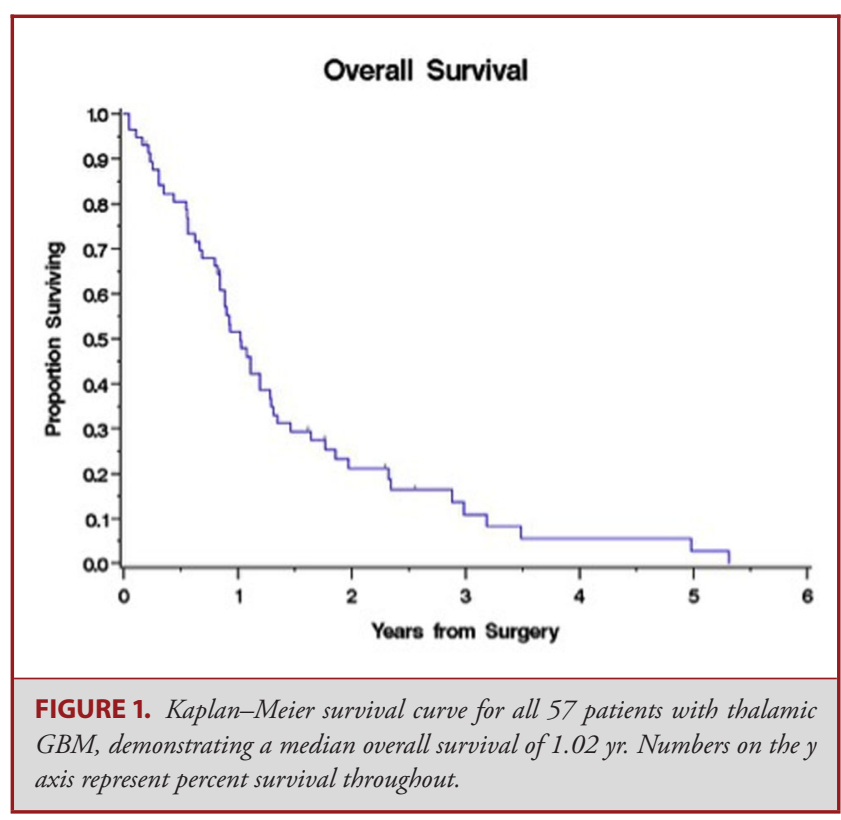

\section{Outcomes}

Median overall OS was $1.02 \mathrm{yr}$ (95\% CI: 0.84-1.28 yr), with $51.5 \%$ and $21.1 \% 1$ - and 2-yr OS, respectively (Figure 1). At the time of study completion, 51 patients had died. There were statistically significant associations of survival with higher KPS (HR [95\% CI] 0.96 [0.94-0.99], $P<.05)$, younger age (HR [95\% CI] 1.02 [1.002-1.03], $P<.05)$, and freedom from language symptoms at presentation on univariate analysis (Table 2). Performance of palliative CSF diversion was also significantly correlated with survival (HR [95\% CI] $0.43[0.23-0.80], P=.008)$. The median OS for patients with KPS $<80$ was 0.75 vs $1.28 \mathrm{yr}$ for those with KPS $>80(P=.008$; Figure $2 \mathrm{~A})$. Younger patients had significantly better OS (HR [95\% CI] 1.02 [1.002-1.042], $P=.03$ ). The median OS of patients presenting with and without language symptoms upon initial diagnosis was 0.88 and $1.11 \mathrm{yr}$, respectively (HR [95\% CI] 2.36 [1.17-4.73], $P=.02$; Figure 2). The median OS in patients with or without ventriculoperitoneal shunt was 1.64 and $0.85 \mathrm{yr}$, respectively (HR [95\% CI] 0.43 [0.23-0.80], $P=.008$; Figure 2C). In multivariate analysis, however, the only significant predictor of survival was KPS (HR [95\% CI] 0.97 [0.95-0.99], $P=.01$ ), although age and CSF diversion remain of clinical significance (Table 2). There was no statistical significant difference in age, KPS, or tumor volume between shunted and nonshunted patients. Shunted patients had higher statistically significant rates of symptoms (headaches, nausea, and vomiting) and radiographic hydrocephalus as well as midbrain involvement.

\section{Radiographic Characteristics and Growth Patterns}

In 53 (93\%) patients, MRI demonstrated features consistent with high-grade tumors such as heterogeneous and/or ring- enhancement with necrosis. ${ }^{16}$ Four cases $(7 \%)$ presented with T2-Flair changes and no or minimal enhancement. Tumors were confined to the thalamus (ventral, medial, lateral, and pulvinar) in $15(28 \%)$ cases. The thalamus and the midbrain (cerebral peduncle, tegmentum, and tectum) were involved in 9 (17\%) cases. While the bulk of the tumor was located in the thalamus in the rest of the cases, in $30(56 \%)$ cases, there was also extension of tumor into adjacent lateral or superior extrathalamic structures such as the periventricular frontal or parietal white matter, mesial temporal structures, the periatrial area, the basal ganglia, or the internal capsule. Bilateral thalamic involvement was present in 3 patients. In 6 cases, tumors extended medially into the third ventricular wall; however, none of these exhibited ependymal violation. Twenty-seven patients $(47 \%)$ presented with radiographic hydrocephalus and $5(9 \%)$ with acute tumoral hemorrhage. Midbrain involvement was seen in 18 patients $(32 \%$; Table 1).

Follow-up imaging ( $>2 \mathrm{mo}$ ) was available in 24 patients $(42 \%)$. In general, tumors growing medially distorted the ventricular wall but did not violate the ependyma, and there was no evidence of intraventricular extension in any case. Tumors growing superiorly displaced and distorted the corpus callosum; ventricular extension was rare (1 case). Laterally and superiorly extending tumors expanded into the subpallial white matter of an adjacent lobe or gyrus such as the frontal and parietal lobes. Tumors restricted to the thalamus tended to remain in the compartment of origin without progression on subsequent imaging, although inferior extension into the midbrain upon progression was seen in a few cases. Thalamic tumors with midbrain involvement remained within the confines of this structure, with local progression in the majority of cases. Bilateral occurrence was observed only rarely (3 cases), as most tumors had unilateral involvement, and none progressed to the contralateral thalamus. CSF dissemination during the course of the disease occurred only in 2 of the 24 patients in whom follow-up imaging was available.

\section{DISCUSSION}

Thalamic glioblastomas are rare, and their natural history and treatment are only infrequently the focus of published literature. ${ }^{5-7,17}$ Due to their deep and eloquent location, these rare tumors are not amenable to extensive surgical resection, removing the option of surgical cytoreduction-one of the few efficacious therapies in glioblastoma - and contributing to unfavorable prognosis. Recent studies have evaluated the role of extent of resection in glioblastoma surgery, most commonly excluding patients with deep-seated tumors, who typically undergo needle biopsy without resection. ${ }^{1,2,18}$ Consequently, data regarding the natural history and prognostic factors in thalamic glioblastoma is lacking. We report the largest series of patients with thalamic glioblastoma described to date. The findings of our study suggest that while these tumors have previously been considered 

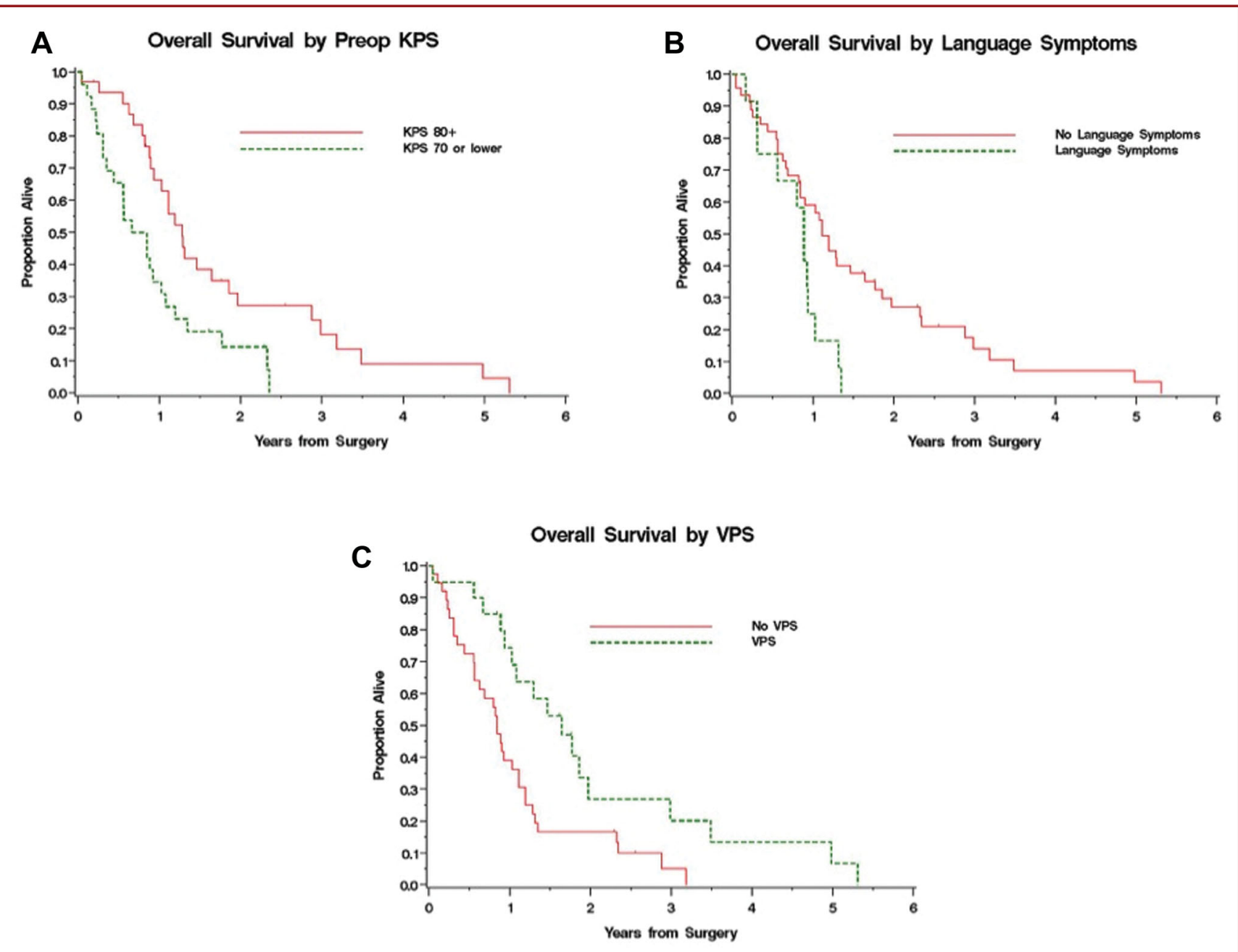

FIGURE 2. A, Kaplan-Meier survival curve representing patients stratified by KPS. Survival was 0.75 and $1.28 \mathrm{yr}$ for patients with KPS $<80$ and $\geq 80$, respectively $(P=.008) . \mathbf{B}$, representing patients with or without presence of language symptoms at presentation, overall survival was 0.88 and $1.11 \mathrm{yr}$, respectively $(P=.02) . \mathrm{C}$, representing patients with or without ventriculoperitoneal shunt during the course of the disease, the overall survival was 1.64 and $0.85 \mathrm{yr}$, respectively $(P=.008)$.

uniformly poor, these patients may have survival outcomes that are comparable to those seen in historic controls of supratentorial lobar glioblastoma who undergo subtotal resection. ${ }^{1-3,19}$ As previously described in GBM studies, we also found that age and KPS were prognostic factors. ${ }^{1,19-21}$ While the association of age with OS is not unexpected, it did not remain significant in the multivariable model in our study.

As expected, and due to their deep location, symptomatology in our cohort was distinct relative to tumors that arise in the cerebral hemispheres. The most common symptoms were motor deficits and/or symptoms of intracranial hypertension, as a consequence of involvement of the motor pathways and CSF obstruction leading to hydrocephalus. Memory loss was also a common symptom. The thalamus represents a major relay center for integrating sensory and motor pathways that originate from distinct cortical and cerebellar regions. It also plays an important role in language and memory, and prior evidence in children with thalamic tumors suggests that specific alterations in neuropsychological profiles might occur with pathology in different thalamic regions. Language comprehension, naming deficits, poor recall, speech apraxia, and oculomotor incoordination have all been described in children with thalamic tumors. ${ }^{22}$ Interestingly, the presence of language symptoms upon diagnosis (naming difficulties, fluency, articulation apraxia, and slowness of speech) was associated with an increased risk of death $(P<.02)$ in our study. Language dysfunction and impaired social interaction are likely contributors of worse functional outcomes, and the deficits that occur as a result of tumor infiltration and mass effect are unlikely to improve following surgical or medical treatment in thalamic tumors. 
While $47 \%$ of patients presented with evidence of obstructive hydrocephalus, only 20 patients $(35 \%)$ underwent cerebrospinal fluid diversion. The majority of these patients had CSF diversion soon after their presentation, and this variable was treated as a baseline covariate. Univariate analysis demonstrated that patients undergoing placement of ventriculoperitoneal shunt have increased survival $(1.64 \mathrm{yr})$ when compared to patients not undergoing CSF diversion $(0.85 \mathrm{yr}, P=.008)$. This finding suggests either a protective effect of CSF diversion or selection bias for patients with small-volume disease or better age/functional status at presentation, although the protective effect of CSF diversion was not significant after controlling for KPS, age, and language symptoms. While no longer statistically significant because of event size (51 deaths), the magnitude and direction of the effect of CSF diversion on OS remained consistent in the multivariable setting. Shunted patients had significantly higher rates of hydrocephalus and midbrain involvement by the tumor, but there was no statistical significant difference in age, KPS, or tumor volume between shunted and nonshunted patients. Considering the cognitive changes that occur following adjuvant therapy and disease progression, the long-term effects of hydrocephalus may be unrecognized during the course of the disease, possibly leading to functional deterioration and shorter survival outcomes. Therefore, a low threshold for CSF diversion may have an impact in those patients in whom even small tumors could obstruct CSF egress, and should be considered.

Although higher complications are known to occur following stereotactic biopsy in deeply located lesions, the complication rate observed in our series (16\%) was higher than prior reports. ${ }^{23,24}$ Despite unremarkable postoperative imaging and lack of tumoral hemorrhage, postoperative deficits can occur following biopsy in deeply located tumors. The close relationship of the thalamus to major functional areas in the setting of tumor edema can lead to neurological deficits following biopsy or surgical debulking. It should also be noted that this complication rate $(16 \%)$ is likely biased favorably, as many other patients may have been treated elsewhere and developed severe complications, making referral to this tertiary care center less likely. While extensive reports are available assessing the role of surgical resection in thalamic and thalamopeduncular lower grade tumors in children, the extent to which resection should be pursued in adult thalamic high-grade gliomas is unclear. ${ }^{25-28}$ Considering that incomplete resection and higher grade histology are known to be poor prognostic factors in thalamic tumors, a more conservative approach with a goal of obtaining histological confirmation followed by prompt and aggressive adjuvant therapy should be considered. $7,25,29$ Ten $(17 \%)$ patients in our cohort underwent craniotomy for attempted resection. Postoperative imaging in patients undergoing craniotomy revealed that, as might be expected, all resections were subtotal. Although a presumptive diagnosis of GBM is likely highly accurate in many cases, it has been our practice to obtain tissue diagnosis whenever feasible, not only for diagnostic purposes but also for molecular analysis and trial eligibility. While we do not embrace a strategy of treatment without tissue confirmation, empiric treatment in this population may not be unreasonable considering recent advances in diagnostic brain tumor imaging ${ }^{30}$ as well as a high rate of complications that can result from a needle biopsy (15\%). While extent of resection remains a key prognostic factor in glioblastoma, we did not find a difference in survival outcomes in patients undergoing stereotactic biopsy vs craniotomy, but this is confounded by the small number of patients and the retrospective nature of our study. Although only a small group of patients underwent surgical debulking, our limited data do not support surgery in the management of thalamic glioblastoma beyond biopsy to obtain a histologic diagnosis. However, 3 patients undergoing surgical resection had large cystic intratumoral components with symptoms of raised intracranial pressure, and 1 patient had a large component of the tumor extending into the frontal lobe
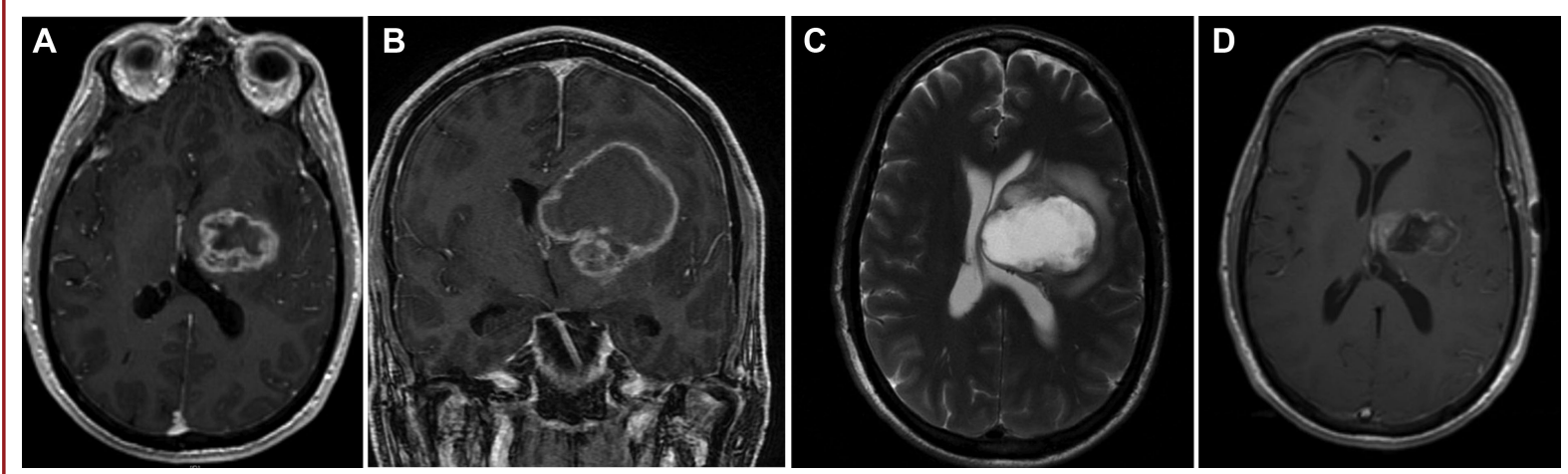

FIGURE 3. A, Axial and B, coronal postcontrast MRI of the brain demonstrating a large heterogeneously enhancing mass with central necrosis and a large cystic component. C, Originating in the left thalamus and expanding into the surrounding frontal white mater. This patient underwent craniotomy due to the large size of the tumorlcystic component. D, Axial postcontrast MRI demonstrates decompression of the cystic and subtotal resection of the enhancing nodular component. 

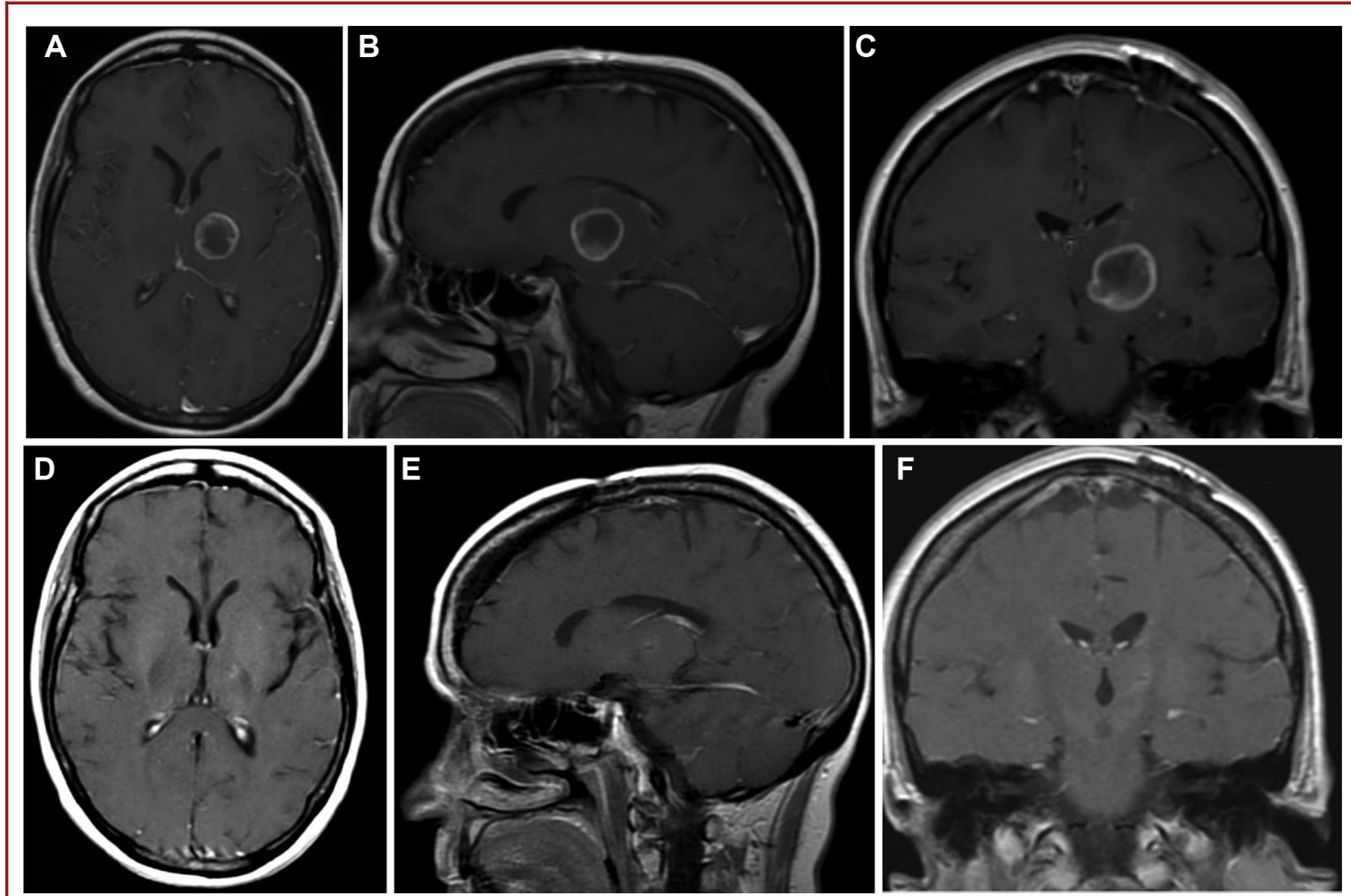

FIGURE 4. Postcontrast axial A, sagittal B, and coronal C MRI of the brain demonstrating a glioblastoma (IDH wild type/MGMT methylated) originating in the left thalamus. This patient underwent stereotactic biopsy and conventional adjuvant chemo-radiation therapy. The tumor remains controlled D-F, and the patient is functional with no evidence of progression 46 mo following initial diagnosis. This tumor is wild type for IDH and MGMT methylated.

(Figure 3). It is possible that these patients with significant mass effect from their large tumors might have had worse outcomes without surgical decompression. Thus, surgical judgment is still important, and in a small number of highly selected patients, some form of resection may be appropriate. While the thalamus can be accessed surgically through several different routes, patients undergoing craniotomy in the current study underwent either a transcortical (transtemporal, 4; transfrontal, 3) or interhemispheric transcallosal approach in the rest. Further surgical and decision-making details for these particular cases are not available, as none of these patients were primarily treated in our institution. A recent study by Cao et $\mathrm{a}^{31}$ evaluated the outcomes of surgical resection in a large series of adult patients with thalamic tumors. The OS of patients with high-grade tumors in this study was 12 mo, which is similar to the findings in our GBM cohort. Of note, high-grade tumors in this study included anaplastic astrocytomas, oligodendrogliomas, oligoastrocytomas, and anaplastic ependymomas, which are known to have a better OS than GBM. Additionally, a perioperative mortality rate of $4.5 \%$ was reported, and transient hemiparesis occurred in about half of the patients. Other complications, such as sensory and visual deterioration, occurred in $15.1 \%$ and $5.7 \%$, respectively, in addition to cerebral edema and speech deterioration, which were not uncommon. Sai Kiran et $\mathrm{al}^{6}$ reported 12 patients with thalamic GBM undergoing aggressive resection. Near total resection was achieved in more than half of them. OS was reported in 3 patients at 1, 1 and 5 mo. The rest of the patients were lost to follow-up, which is likely a reflection of the poor postoperative outcome in these cases. Steiger et a ${ }^{32}$ reported 14 cases of thalamic astrocytomas in which maximum microsurgical removal was performed. Three of the cases were GBMs, and in 1 case a postoperative visual deficit occurred. One patient died 4 mo following surgery, and the follow-up for the other 2 cases was limited to 6 and 10 mo. The results of these studies suggest that aggressive resection of thalamic glioblastoma may be questionable considering the aggressive nature of the disease and the limited time that might be available for rehabilitation prior to initiation of adjuvant therapy. Future advances in surgical technology, coupled with intraoperative neurophysiological monitoring, might improve the risk profile of surgical interventions in the thalamus, although the function and anatomic location of this area will remain as inherent challenges. Laser interstitial thermal ablation, a current subject of clinical trials, is another alternative that can be performed after stereotactic biopsy and should be considered. 


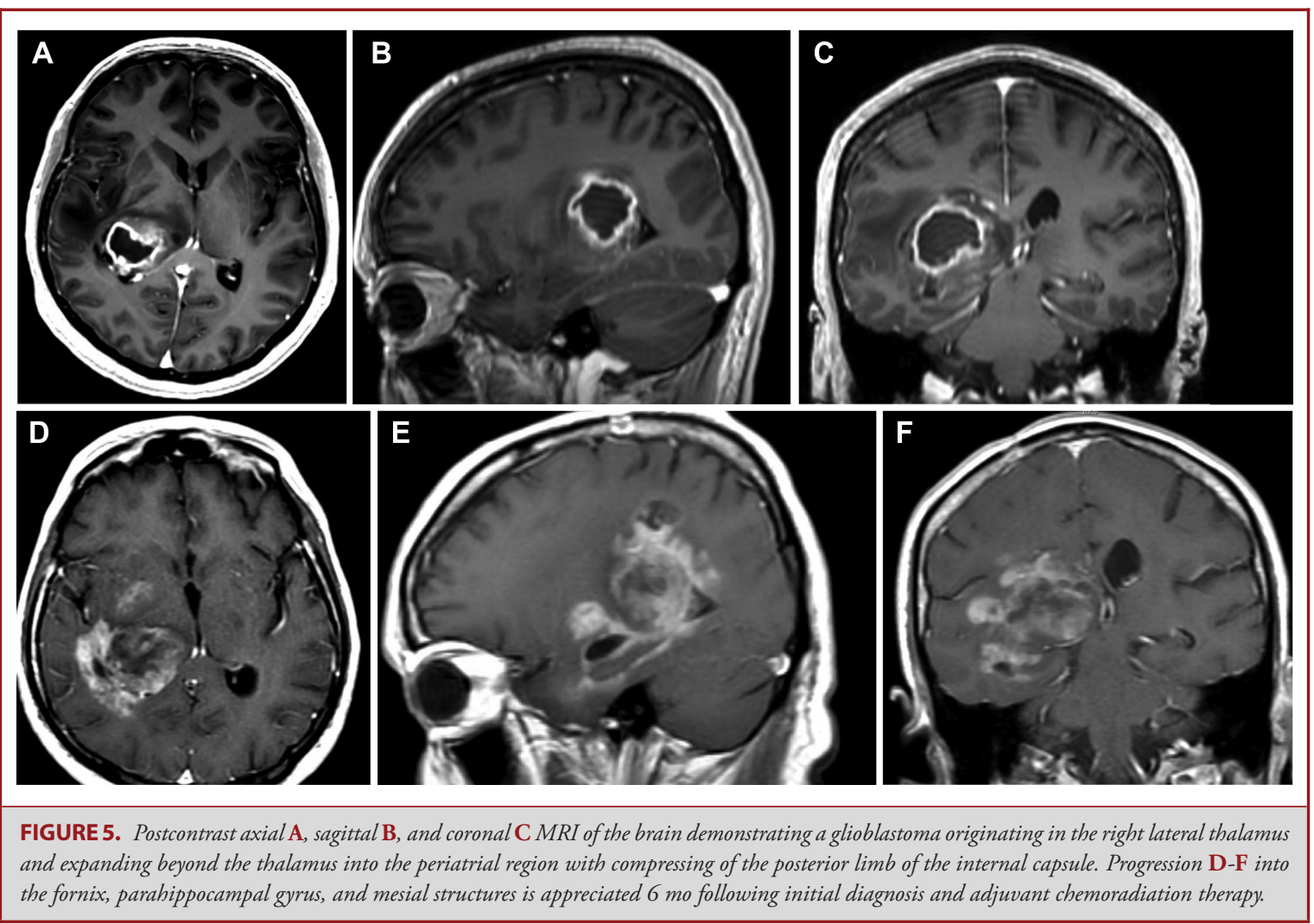

Recent genomic analyses have revealed that specific genetic variations drive distinct subgroups of glial neoplasms and depend not only on the tumor type but also on the patient age and site of origin. Novel histone mutations in thalamic tumors have been described, but with a high preponderance in children and young adults. ${ }^{33,34}$ Because molecular analysis is lacking in this study, it is unclear whether the genomic profile of the tumors plays a significant role in their outcome. Studies have revealed that diffuse midline gliomas with histone H3-K27M mutation have an aggressive clinical behavior and poor prognosis; however, recent studies have also shown that thalamic gliomas in adult patients with histone $\mathrm{H} 3-\mathrm{K} 27 \mathrm{M}$ mutation might not be associated with worse prognosis than histone $\mathrm{H} 3$ wild-type thalamic gliomas. ${ }^{12,33-36}$ This suggests heterogeneity within this molecular subgroup, and larger studies should be performed to evaluate the efficacy of histone-modifying enzymes as potential treatment options in adult thalamic glioblastoma. ${ }^{10,37,38}$

Age and KPS were key criteria that impacted survival in this study, a pattern that is similar to that seen in hemispheric gliomas. ${ }^{19,20}$ Adding to the fact that thalamic gliomas do not undergo a significant debulking, outcomes of thalamic tumors seem indeed to follow the general pattern of lobar glioblastomas. Considering the deep and eloquent location of these tumors in which resection would lead to significant neurological deficits, novel technologies such as laser interstitial thermal ablation aimed at maximal local cytoreductive therapy could potentially have a role in a select group of patients (tumors smaller than $3 \mathrm{~cm}$ ) and should be evaluated. ${ }^{39}$

\section{Patterns of Progression}

Similar to Yasargil's ${ }^{40}$ observations, tumor enlargement demonstrated displacement of the surrounding structures such as the internal and external capsules and adjacent nuclei, although, despite growth, they respected their anatomic fiber tract borders during the initial stage of the tumor. Prior studies using virtual tumor modeling have demonstrated that glioma cells migrate slower in grey matter than in white matter, perhaps consistent with our radiographic observations in many cases in which tumors remained localized for a long period of time. ${ }^{41}$ This was mostly seen among patients with tumors restricted to the thalamus, which tended to remain in the compartment of origin for extended periods of time (Figure 4) as opposed to patients with tumors involving white matter structures at presentation that progressed rapidly (Figure 5). There was no statistical significant difference in OS between patients with or without midbrain involvement. These findings suggest that tumor extension into the midbrain should not be considered a worse prognostic factor, as survival in these patients does not appear to be worse than tumors without brainstem involvement. It is important to clarify that in view of the limited number of patients with follow-up 
imaging, strong conclusions about patterns of recurrence cannot be drawn.

\section{Limitations}

This study is limited by its retrospective design and the fact that the majority of patients $(88 \%)$ were not managed primarily at our institution. Additionally, we were unable to analyze the role of chemotherapy on survival, as adequate treatment data were not available. ${ }^{42}$ Molecular profiling for clinically relevant parameters in GBM, including isocitrate dehydrogenase and histone H3F3A K27 genotyping, known to drive a subset of pediatric high-grade gliomas, as well as epigenetic changes such as $\mathrm{O}^{6}$-methylguanineDNA methyltransferase status, was only rarely available. ${ }^{43,44} \mathrm{Lack}$ of long-term imaging data precluded growth pattern analysis for many patients.

\section{CONCLUSION}

This study represents a large and unique series of patients with thalamic glioblastoma. The OS of these patients is comparable to historic data of incompletely resected supratentorial lobar glioblastoma. Younger patients and those with improved initial functional status (KPS $>80$ ) have better outcomes. CSF diversion should be considered early in patients with symptomatic or radiographic hydrocephalus. Midbrain involvement by the tumor is not in and of itself a negative prognostic factor. In the setting of high morbidity and mortality, improved therapies are needed, and patients should be considered for early trial involvement and upfront aggressive therapies upon histological confirmation. The results of our study may serve as benchmarks for the natural history of thalamic glioblastoma and can be useful to compare outcomes in patients in whom novel strategies are employed. Future study is needed to further characterize thalamic glioblastoma molecularly and to develop more effective therapies for this patient population.

\section{Disclosures}

This work was funded by Core Grant P30 CA008748. The authors have no personal, financial, or institutional interest in any of the drugs, materials, or devices described in this article.

\section{REFERENCES}

1. Sanai N, Polley MY, McDermott MW, Parsa AT, Berger MS. An extent of resection threshold for newly diagnosed glioblastomas. J Neurosurg. 2011;115(1): 3-8.

2. Orringer D, Lau D, Khatri S, et al. Extent of resection in patients with glioblastoma: limiting factors, perception of resectability, and effect on survival. J Neurosurg. 2012;117(5):851-859.

3. McGirt MJ, Chaichana KL, Gathinji M, et al. Independent association of extent of resection with survival in patients with malignant brain astrocytoma. J Neurosurg. 2009;110(1):156-162.

4. Esquenazi Y, Friedman E, Liu Z, Zhu JJ, Hsu S, Tandon N. The survival advantage of "supratotal" resection of glioblastoma using selective cortical mapping and the subpial technique [published online March 23, 2017]. Neurosurgery. 2017;81(2), doi: 10.1093/neuros/nyw174.
5. Grigsby PW, Garcia DM, Simpson JR, Fineberg BB, Schwartz HG. Prognostic factors and results of therapy for adult thalamic and brainstem tumors. Cancer. 1989;63(11):2124-2129.

6. Sai Kiran NA, Thakar S, Dadlani R, et al. Surgical management of thalamic gliomas: case selection, technical considerations, and review of literature. Neurosurg Rev. 2013;36(3):383-393.

7. Nishio S, Morioka T, Suzuki S, Takeshita I, Fukui M. Thalamic gliomas: a clinicopathologic analysis of 20 cases with reference to patient age. Acta Neurochir (Wien). 1997;139(4):336-342.

8. Buczkowicz P, Hawkins C. Pathology, Molecular genetics, and epigenetics of diffuse intrinsic pontine glioma. Front Oncol. 2015;5:147.

9. Zhang R, Han J, Daniels D, Huang H, Zhang Z. Detecting the H3F3A mutant allele found in high-grade pediatric glioma by real-time PCR.J Neurooncol. 2016;126(1):27-36.

10. Solomon DA, Wood MD, Tihan T, et al. Diffuse midline gliomas with histone H3-K27M mutation: a series of 47 cases assessing the spectrum of morphologic variation and associated genetic alterations. Brain Pathol. 2016;26(5):569-580.

11. Hayashi Y, Yamashita J, Watanabe T. Molecular genetic analysis of deep-seated glioblastomas. Cancer Genet Cytogenet. 2004;153(1):64-68.

12. Aihara K, Mukasa A, Gotoh K, et al. H3F3A K27M mutations in thalamic gliomas from young adult patients. Neuro Oncol. 2014;16(1):140-146.

13. Tovi D, Schisano G, Liljeqvist B. Primary tumors of the region of the thalamus. J Neurosurg. 1961;18:730-740.

14. Cheek WR, Taveras JM. Thalamic tumors. J Neurosurg. 1966;24(2):505-513.

15. Louis DN, Ohgaki H, Wiestler OD, et al. The 2007 WHO classification of tumours of the central nervous system. Acta Neuropathol. 2007;114(2):97-109.

16. Kurian KM, Zhang Y, Haynes HR, Macaskill NA, Bradley M. Diagnostic challenges of primary thalamic gliomas-identification of a minimally enhancing neuroradiological subtype with aggressive neuropathology and poor clinical outcome. Clin Neuroradiol. 2014;24(3):231-238.

17. Pathy S, Jayalakshmi S, Chander S, Singh R, Julka PK, Rath GK. Prognostic factors influencing the outcome of thalamic glioma. Neurol India. 2002;50(1): $37-40$.

18. Omuro A, DeAngelis LM. Glioblastoma and other malignant gliomas: a clinical review. JAMA. 2013;310(17):1842-1850.

19. Lacroix M, Abi-Said D, Fourney DR, et al. A multivariate analysis of 416 patients with glioblastoma multiforme: prognosis, extent of resection, and survival. J Neurosurg. 2001;95(2):190-198.

20. Buckner JC. Factors influencing survival in high-grade gliomas. Semin Oncol. 2003;30(6 suppl 19):10-14.

21. Chaichana KL, Halthore AN, Parker SL, et al. Factors involved in maintaining prolonged functional independence following supratentorial glioblastoma resection. Clinical article. J Neurosurg. 2011;114(3):604-612.

22. Chieffo D, Tamburrini G, Caldarelli M, Di Rocco C. Preoperative neuropsychological and behavioral evaluation of children with thalamic tumors. J Neurosurg Pediatr. 2014;13(5):507-513.

23. McGirt MJ, Woodworth GF, Coon AL, et al. Independent predictors of morbidity after image-guided stereotactic brain biopsy: a risk assessment of 270 cases. J Neurosurg. 2005;102(5):897-901.

24. Kelly PJ. Stereotactic biopsy and resection of thalamic astrocytomas. Neurosurgery. 1989;25(2):185-194; discussion 194-195.

25. Puget S, Crimmins DW, Garnett MR, et al. Thalamic tumors in children: a reappraisal. J Neurosurg. 2007;106(5 suppl):354-362.

26. Kramm CM, Butenhoff S, Rausche U, et al. Thalamic high-grade gliomas in children: a distinct clinical subset? Neuro Oncol. 2011;13(6):680-689.

27. Wisoff JH, Boyett JM, Berger MS, et al. Current neurosurgical management and the impact of the extent of resection in the treatment of malignant gliomas of childhood: a report of the Children's Cancer Group trial no. CCG-945. JNeurosurg. 1998;89(1):52-59.

28. Bilginer B, Narin F, Isikay I, Oguz KK, Soylemezoglu F, Akalan N. Thalamic tumors in children. Childs Nerv Syst. 2014;30(9):1493-1498.

29. Eisenstat DD, Pollack IF, Demers A, et al. Impact of tumor location and pathological discordance on survival of children with midline high-grade gliomas treated on Children's Cancer Group high-grade glioma study CCG-945. J Neurooncol. 2015;121(3):573-581.

30. Moton S, Elbanan M, Zinn PO, Colen RR. Imaging genomics of glioblastoma: biology, biomarkers, and breakthroughs. Top Magn Reson Imaging. 2015;24(3):155-163. 
31. Cao L, Li C, Zhang Y, Gui S. Surgical resection of unilateral thalamic tumors in adults: approaches and outcomes. BMC Neurol. 2015;15:229.

32. Steiger HJ, Gotz C, Schmid-Elsaesser R, Stummer W. Thalamic astrocytomas: surgical anatomy and results of a pilot series using maximum microsurgical removal. Acta Neurochir(Wien). 2000;142(12):1327-1336; discussion 1336-1337.

33. Korshunov A, Ryzhova M, Hovestadt V, et al. Integrated analysis of pediatric glioblastoma reveals a subset of biologically favorable tumors with associated molecular prognostic markers. Acta Neuropathol. 2015;129(5):669-678.

34. Khuong-Quang DA, Buczkowicz P, Rakopoulos P, et al. K27M mutation in histone H3.3 defines clinically and biologically distinct subgroups of pediatric diffuse intrinsic pontine gliomas. Acta Neuropathol. 2012;124(3):439-447.

35. Feng J, Hao S, Pan C, et al. The H3.3 K27M mutation results in a poorer prognosis in brainstem gliomas than thalamic gliomas in adults. Hum Pathol. 2015;46(11):1626-1632.

36. Sturm D, Witt H, Hovestadt V, et al. Hotspot mutations in H3F3A and IDH1 define distinct epigenetic and biological subgroups of glioblastoma. Cancer Cell. 2012;22(4):425-437.

37. Grasso CS, Tang Y, Truffaux N, et al. Functionally defined therapeutic targets in diffuse intrinsic pontine glioma. Nat Med. 2015;21(7):827-827a.

38. Hashizume R, Andor N, Ihara Y, et al. Pharmacologic inhibition of histone demethylation as a therapy for pediatric brainstem glioma. Nat Med. 2014;20(12):1394-1396.

39. Hawasli AH, Kim AH, Dunn GP, Tran DD, Leuthardt EC. Stereotactic laser ablation of high-grade gliomas. Neurosurg Focus. 2014;37(6):E1.

40. Yaşargil MG. Microneurosurgery. Volume IVA: CNS tumors: Surgical Anatomy, Neuropathology, Neuroradiology, Neurophysiology, Clinical Considerations, Operability, Treatment Options. New York: Thieme Stratton; 1994:139-142.

41. Swanson KR, Alvord EC, Jr, Murray JD. Virtual brain tumours (gliomas) enhance the reality of medical imaging and highlight inadequacies of current therapy. $\mathrm{BrJ}$ Cancer. 2002;86(1):14-18.

42. Anderson JR, Cain KC, Gelber RD. Analysis of survival by tumor response. J Clin Oncol. 1983;1(11):710-719.

43. Hegi ME, Diserens AC, Gorlia T, et al. MGMT gene silencing and benefit from temozolomide in glioblastoma. $N$ Engl J Med. 2005;352(10):997-1003.

44. Parsons DW, Jones S, Zhang X, et al. An integrated genomic analysis of human glioblastoma multiforme. Science. 2008;321(5897):1807-1812.

\section{COMMENT}

7 he authors review outcomes of 57 patients with thalamic glioblastomas over an approximately 10 -year period. This is an important study to help better understand the natural history and response to therapy of glioblastomas that involve the thalamus.

The authors in general recommend biopsy in all cases for diagnostic purposes, but also for molecular analysis and clinical trial eligibility. Certainly, one must take with caution the recommendation for any type of surgery for patients with suspected thalamic glioblastoma, as the authors report that 7 (15\%) of the 47 patients that underwent stereotactic biopsy suffered fixed neurological deficits, and additionally, 2 (20\%) of the 10 patients that underwent craniotomy suffered new permanent deficits. In some circumstances, I believe that empiric treatment based upon imaging alone, particularly for hypervascular lesions, may be reasonable.

Higher Karnofsky Performance Scores (KPS), younger age, and cerebrospinal fluid (CSF) diversion were correlated with better overall survival with univariate analysis, while the presence of language deficits at initial presentation was associated with poorer survival. Only KPS was predictive of survival in the multivariate analysis. The significance of CSF diversion was not a significant predictor of survival in the based on multivariate analysis, but perhaps trended towards significance. The authors suggest that CSF shunting may be an independent predictor of better outcome, and the neurosurgeon managing patients with thalamic glioblastoma therefore should consider whether to pursue a shunt.

The authors' analyses of outcomes for patients with glioblastomas involving the thalamus serve as a new benchmark for comparison as new treatments such as laser interstitial thermal ablation (LITT) and other systemic therapies are explored. The issue of which tumors should be deemed suitable for surgical resection even with minimally invasive tubular retractors for example, or for stereotactic biopsy, or perhaps even for empiric treatment without tissue diagnosis remains unsolved.
Michael R. Chicoine

St. Louis, Missouri 